\title{
Krise, Kritik und Szenarien: Zur Lage der Demokratie
}

Die Euphorie des Jahrhundertbeginns, auch die des Arabischen Frühlings, ist verflogen. Mehr noch: An ihre Stelle ist Skepsis, Kritik und auch eine gewisse Ratlosigkeit getreten. Man könnte meinen, der Triumph der demokratischen Ordnung über die Systeme des realexistierenden Sozialismus habe sich als Pyrrhussieg entpuppt. Die Zahl der Demokratien auf der Welt ist angewachsen, die Klagen über ihren Zustand auch. Es ist vor allem die innere Entwicklung der etablierten Demokratien, von denen man früher als denen des ,westlichen Typs“ gesprochen hat, die vielfältigen Anlass bietet, von einer Krise der Demokratie zu sprechen. Für manche ist die Demokratie in einen post-demokratischen Zustand der Hegemonie von (Finanz-)Wirtschaft, Medien und Politik der Eliten eingetreten, ganz so, als sei das Zeitalter der Demokratien bereits zu Ende. Andere wiederum sehen die Demokratien in einer autoritären Transformation mit einem populistischen Unterbau. Und, nicht zuletzt durch die europäische Verschuldungs- und Währungskrise deutlich befördert, mutieren Demokratien zur Herrschaft von Experten, einer Form der Technokratie, die sich, mit Carl Schmitt gesprochen, zum globalen Nomos der Erde aufzuschwingen scheint und das Politische durch das Exekutivische ersetzt. Nun ist zwar die Rede über die Krisen der Demokratie fast so alt wie die Demokratie selbst. Doch gibt es derzeit beunruhigende Krisenphänomene und Tendenzen, die das uns bekannte Bild der Demokratie verändern und wieder einmal nach der Zukunft der Demokratie fragen lassen.

Dabei muss man nicht den Alarmismen der Krisendiagnostiker unterschiedlicher theoretischer Provenienzen folgen, die die gegenwärtige Demokratie als halbierte und entparlamentarisierte, als entpolitisierte und unpolitische kennzeichnen und die glauben, dass es ihr an Vitalität und Agonalität mangelt. Vielfach scheinen hier Rückprojektionen vermeintlich goldener Zeitalter, normative Modellierungen und simplizistische Reduktionismen durch, auf deren Hintergrund eine jede konkrete Praxis demokratischer Ordnung defizitär erscheinen muss. Gegen diese verklärenden oder überfrachteten Bilder kann die jeweilige demokratische Gegenwart ohnehin nur öde und fad wirken, das große Versprechen der Demokratie weicht großer Enttäuschung. Aber auch ein realistischer, historisch informierter Blick kommt 
nicht umhin, einen Befund auszustellen, der zu einem neuen Nachdenken über die Demokratie, wie wir sie kennen und wie sie sich derzeit wandelt, führt.

Es geht um Prozesse, die in den Kern des Selbstverständnisses repräsentativer Demokratie zielen. ${ }^{1}$ Mit repräsentativer Demokratie ist ein Typus von Demokratie gemeint, der sich historisch infolge der Revolutionen des 18. Jahrhunderts herausgebildet hat und der auf vermittelnden, stellvertretenden Formen der Entscheidungsbildung basiert. Ein ausgeklügeltes institutionelles Arrangement politischer Ordnung hat den Willensbildungs- und Entscheidungsprozess auf verschiedenen Ebenen organisiert und dabei weniger die direkte Beteiligung der Bürger - jenseits von Wahlen - als vielmehr die stellvertretende Erledigung von Entscheidungs- und Kontrollaufgaben in einem Set wechselseitig aufeinander einwirkender Institutionen präferiert. Ein komplexes Institutionensystem sucht einer Demokratie der großen Zahl und des großen Raumes Stabilität, Legitimität und Effizienz zu geben. Dieses System kunstvoll verfasster institutioneller Ordnung befindet sich offenkundig in einer Krise, deren Ausmaß noch kaum erkennbar und deren Lösungsmöglichkeiten völlig unklar sind. Damit stellt sich die Frage nach den Gründen, die sich als Strukturprobleme der repräsentativen Demokratie zu Beginn des 21. Jahrhunderts beschreiben lassen.

Erstens scheint das System der stellvertretenden Entscheidungsbildung zu komplex und intransparent geworden zu sein. Die gewaltenteilige und, wo vorhanden, föderale Organisation des demokratischen Entscheidungssystems, ihre Einbindung in supranationale Organisationen und internationale Governance-Strukturen führt zu einer komplexen, verschachtelten Struktur von Willens- und Entscheidungsbildung, die kaum den demokratischen Grundsätzen der Transparenz und der klaren Zurechnung von Verantwortlichkeiten genügt. Zahlreiche Spieler, Institutionen, Akteure und Interessengruppen sind in der Lage, den Prozess der komitologischen Aushandlung von Lösungen undurchschaubar zu machen und ihn, zugleich unsichtbar für das demokratische Publikum, zu steuern. Verhandlungssysteme dieser Art mögen, wenn sie zu Lösungen kommen, für die Beteiligten befriedende, auch effektive Ergebnisse einbringen. Aber sie benachteiligen, und zwar in struktureller Weise, solche Gruppen und Individuen, die nicht die Macht oder die Organisationsfähigkeit ihrer Interessen besitzen und deshalb kein Gehör finden. Insofern werden hier Teilhabechancen verwehrt, Publizitätspflichten verletzt und Responsivitätserfordernisse missachtet, die zum Kern demokratischer Ordnungsvorstellungen gezählt werden müssen.

1 Einige der folgenden Überlegungen sind früheren Beiträgen (Vorländer 2011 a und 2011 b) entnommen. Sie sind hier wesentlich überarbeitet, erweitert und akzentuiert worden. 
Zweitens hat die Globalisierung die politischen Entscheidungsstrukturen grundlegend verändert. Die ökonomischen, technologischen und kommunikativen Prozesse halten sich immer weniger an die mehr oder minder künstlichen Grenzen von (National-)Staaten. Damit wachsen auch die Probleme und der politische Regelungsbedarf in den überstaatlichen Bereichen hinein. Global vernetzte Ökonomien eröffnen einen eigenständigen transnationalen Raum, in dem Politik weitestgehend durch Kooperation zwischen Regierungen stattfindet. Diese Formen des Regierens jenseits des demokratischen Nationalstaates ${ }^{2}$ sind aber ohne eine direkte demokratische Legitimation. Deshalb verliert die ursprünglich territorial gebundene und begründete parlamentarisch-repräsentative Demokratie an Substanz, während die Gestaltungsmacht transnationaler Politik ohne direkte demokratische Legitimation anwächst. Die Demokratie befindet sich in einem Dilemma: Wo die Demokratie auf ihrer territorialstaatlichen Verfassung besteht, verliert sie an Regelungseffizienz. Wo die Politik den transnationalen Akteuren in die Globalität folgt, begibt sie sich ihrer territorialstaatlich rückgebundenen Legitimität. ${ }^{3}$ Das Spiel zwischen den Ebenen aber findet ohne den Bürger statt. Diese Diagnose ist nicht neu, aber die Euround Bankenkrise spitzt diese Spannungslage zwischen notwendiger Transnationalisierung der Regulierung und nationalem Legitimationserfordernis dramatisch zu.

Drittens hat sich der öffentliche Raum der Meinungs- und Willensbildung verändert. Zum einen besitzen Massenmedien große öffentliche Deutungsmacht, bestimmen sie die politische Agenda, zum anderen bedient sich die Politik der Massenmedien, um Einfluss auf das (Wähler-)Publikum zu nehmen. Auf mediale Präsenz kommt es in jedem Fall an. Politik wird, wo sie sich der Darstellungsformen des populären Unterhaltungsgenres bedient, zur (Talk) Show, Politik und Entertainment werden ununterscheidbar, sie verschmelzen zu Politainment. ${ }^{4}$ Die Mediendemokratie findet das ihr gemäße Korrelat in der ,Zuschauerdemokratie“: Der Bürger wird zum „Zuschauer der Worte und Hörer der Taten“, wie schon Thukydides Kleon sagen ließ, damit aber den Vorbildcharakter der dramaturgischen Inszenierung von Erzählungen und Heroen betonte. Davon kann heute, wegen der Entzauberung der Narrative und der beschleunigten Taktung medialer Berichterstattung, nicht mehr die Rede sein. Die modernen audiovisuellen Leitmedien machen eine Bilder- und Formensprache verbindlich, die auf kurzzeitige Aufmerksamkeitsgewinne programmiert ist. Politik wird auf eine Logik des Darstellbaren und Inszenatorischen festgelegt, hinter der die politischen Beratungs-, Kompro-

\footnotetext{
2 Siehe Zürn 1998.

3 Vergleiche Beck 1998.

4 Hierzu Dörner 2001.
} 
missbildungs- und Entscheidungsverfahren notwendig zurückbleiben müssen: Was sich nicht visualisieren und inszenieren lässt, entgeht dem Wahrnehmungshorizont des Zuschauers, ist deshalb nicht existent. Die Dramatologie des Visuellen unterläuft die Komplexität des Politischen.

Zugleich verstärken sich durch die elektronischen Medien, vor allem das Internet und die sozialen Medien, die Formen anonymer und medial vermittelter Kommunikation unter Abwesenden, die aber jenen verdichteten Kommunikationszusammenhang kaum herzustellen vermögen, der für unmittelbare Bürgerbeteiligung und bürgernahe Entscheidungsverfahren notwendig ist. Die vielfach bemühte $e$-democracy - elektronische oder digitale Demokratie - erhöht zwar die Beteiligungs- und Zugangschancen, indem es interessierten Bürgern Informations- und Bildungsangebote, Kommunikations- und Organisationsmöglichkeiten in einem zuvor nicht gekannten Ausmaß bereitstellt, doch schafft Online-Kommunikation nicht per se eine breitere oder gar bessere politische Öffentlichkeit. Wir wissen mittlerweile, dass die digitale Demokratie die sozialen Diskriminierungs- und Selektionsmechanismen, die den traditionellen Partizipationsformen zueigen sind, repliziert. Damit dementieren sich auch die Transparenzbehauptungen, liquider Demokratie', die die digitale Utopie von der Beteiligung Aller an Allem zu realisieren hoffte, dann aber, nach der Erfahrung eines - physisch wie psychisch - unbeherrschbaren Kommunikationsflusses, in mehr oder weniger orthodoxen Repräsentativstrukturen endete.

Viertens hat sich die politisch-soziale Infrastruktur zeitgenössischer Demokratie grundlegend gewandelt. Die Prägekraft von sozialen Milieus, intermediären Einrichtungen und politischen Vorfeldorganisationen hat nachgelassen. Gleichzeitig privilegieren die neuen sozialen Medien situative Interventionsmodi, von schwarmintelligenten Artikulations- und Protestformen bis zu eruptiven Aufwallungen politischer Stimmungslagen. Beide Befunde, das Fehlen wirksamer Bindungen und der neue Erregungs- und Empörungsstil des Politischen, führen zu immer neuen Adhocismen, einem Politikstil des Sofortigen und Unmittelbaren, der als Reaktion auf Krisen zwar angemessen, manchmal sogar ,alternativlos“ zu sein scheint, auf die Dauer aber das Vertrauen in die Rationalität demokratischer Willens- und Entscheidungsbildung untergräbt.

Fünftens erzeugt die zeitgenössische Demokratie interne Spannungslagen, die zwar in ihr angelegt sind, nun aber, unter Stress, belastend wirken und Kosten entweder auf Seiten der Effizienz oder auf Seiten der Legitimation oder auf beiden Seiten verursachen. Ein hohes - wenngleich in seinen konkreten Formen mitunter diffuses - Partizipationsbegehren erfordert einen anderen Zeithorizont, als es schnelle Entscheidungsnotwendigkeiten - besonders in Krisensituationen - zulassen. Die konstitutionelle Demokratie stattet die (Verfassungs-)Judikative mit Vor- 
rang in der Kontrolle von Institutionen, Prozeduren und Gesetzen aus, was, wie im bundesdeutschen Fall, zu einer Legitimationsverschiebung im demokratischen Institutionengefüge führen kann. Wo der Bürger in seiner gewachsenen Skepsis gegenüber repräsentativ verfasster Politik Zuflucht zu ,gegendemokratischen“ Formen politischen Handelns, von Protest, Besetzung öffentlicher Plätze bis zu rechtlichen Interventionen nimmt, ${ }^{5}$ wird es zunehmend schwieriger, überhaupt noch Entscheidungen zu fällen und durchzusetzen. Wo sich die Vitalität des demokratischen Momentes nur mehr in Eruptionen von „Volkes Stimme“, aber nicht mehr als Responsivität des institutionalisierten Verfahrens Bahn bricht, delegitimiert sich das Repräsentative, und das sowohl auf der Input- wie der Outputseite.

Die Krisenphänomene und Strukturprobleme der zeitgenössischen Demokratie sind leichter zu identifizieren als Heilmittel anzugeben, mittels derer die Gefährdungen zu beheben sind. Gegenwärtige Demokratietheorien sind hier auch nicht wirklich weiterführend. Der nostalgische Blick auf vermeintlich goldene Zeitalter der (Nachkriegs-)Demokratie, in dem fundamentale gesellschaftliche Konflikte einen lebendigen, unverstellten, agonalen Wettbewerb der Ideen, Parteien und Klassen erzeugt hätten, ${ }^{6}$ entspringt eher dem Ennui einer sich heute depossediert fühlenden Klasse professioneller und akademischer Deuter als einer historisch zutreffenden Analyse der seinerzeit als eng, unemanzipiert und ,spießbürgerlich' empfundenen 1950er und 1960er Jahre. Radikale Theorien ihrerseits scheinen angesichts einer offensichtlich depolitisierten und unpolitisch gewordenen Demokratie den Momenten demokratischer Ursprünglichkeit, einer Manifestation der Souveränität des Demos, die die im Zeichen des „Neoliberalismus“ errichtete Herrschaft von Wirtschaft, Medien und Politik beseitigt, entgegenzufiebern. ${ }^{7}$ Und deliberative Demokratiekonzeptionen haben sich in den letzten Jahrzehnten im Zeichen eines „epistemischen Prozeduralismus“ (Estlund), der weniger das Ausmaß der Partizipation als die faktisch selektive Zugänglichkeit zum deliberativen Prozess ins Auge fasste, so weit selbst diszipliniert, dass sie schon auf der Seite der „Depolitisierer“ verbucht werden, weil sie die „Entkoppelung der [...] Entscheidungsträger nicht mehr als demokratisches Übel, sondern als deliberativen Mehrwert" (Höreth 2009: 325) zu verkaufen wissen. Ironischerweise wird hier die Vernünftigkeit der in Institutionen oder Netzwerken deliberativ generierten Dezision just in dem Moment konzediert, wo die Institutionen der stellvertretenden, repräsentativen Willens- und Entscheidungsbildung in eine Vertrauenskrise geraten.

5 Vergleiche auch Rosanvallon 2006.

6 So beispielsweise Crouch 2008.

7 So beispielsweise Rancière 2002. Anders, auf die Wiedergewinnung des agonalistischen Moments setzend, Mouffe 2008. 
Im Grunde ist die Situation paradox: Die Klage über den gegenwärtigen Zustand der Demokratie korreliert mit einem bemerkenswerten Revival der Demokratietheorie. ${ }^{8}$ Aber die Perspektiven auf eine Besserung der Lage sind merkwürdig altbacken, sie reichen von Forderungen nach stärkerer Beteiligung, höherer Transparenz, Wiederherstellung der Konfliktfähigkeit bis hin zur Wiedereinsetzung des Demos in seinen revolutionären Stand. Vielleicht sind auch die Diagnosen verkehrt, nicht gründlich genug, weil sie auf theoretischen Annahmen und Gesellschaftsbeschreibungen beruhen, die den Blick auf sich gegenwärtig vollziehende gesellschaftliche Transformationen verstellen, die die politisch-kulturellen Voraussetzungen von Demokratien tangieren und die sich selbst womöglich nur als Paradoxien beschreiben lassen. Ausgehend von der Feststellung, dass die Demokratie „mitnichten tot, sondern im Gegenteil sehr lebendig ist“" (Blühdorn 2013 b: 134), einer Beschreibung, der mit Blick auf die Vielfalt zivilgesellschaftlicher Aktivitäten kaum zu widersprechen ist, ${ }^{9}$ kommt Ingolfur Blühdorn zu der bemerkenswerten These, dass sich die gegenwärtige Demokratie am ehesten als ,simulative Demokratie" (Blühdorn 2013 a) beschreiben lässt. Damit ist die Bewältigungsstrategie einer paradoxen Situation gemeint, die darin besteht, dass zwar die demokratischen Grundnormen uneingeschränkt gültig sind und im politischen Diskurs beschworen werden, andererseits aber die Bereitschaft und die Fähigkeit abnehmen, ,sich von den Normen der Demokratie in die Pflicht nehmen zu lassen“ (ders. 2013 b: 135). Dies betrifft nicht nur die wirtschaftliche Macht und die politisch-administrativen Eliten, sondern auch andere gesellschaftliche Akteure, denen demokratische Normen gleichermaßen „lästig wie unverzichtbar“ (ebd.: 136) sind. Verstetigtes demokratisches Engagement verursacht Kosten, die aber unter Effizienzgesichtspunkten nicht mehr tragbar erscheinen. Insofern befreie sich auch das demokratische und autonome Subjekt von den demokratischen Zumutungen und orientiere Selbsterfahrung und Identitätsbildung an den Leitmedien von Markt und Konsum. Demnach ist die gegenwärtige - ,postdemokratische“ - Konstellation nicht nur das Ergebnis von wirtschaftlicher, medialer und administrativer Macht, sondern auch eines grundlegenden gesellschaftlichen Wandels, der dafür sorgt, dass sich Bedürfnisse viel besser über den Markt als durch die Demokratie befriedigen lassen. Die „simulative Demokratie“ hat nach Blühdorn deshalb ,ihren Ort in einer Vielzahl kommunikativer und politisch-administrativer Verfahrensweisen, die bei Bedarf als Be-

8 Vergleiche jetzt die sehr anregende Studie von Michelsen/Walter 2013, die - dramaturgisch geschickt - bisweilen das Krisenlamento rhetorisch überziehen, dann aber in der Sache selbst erfrischend nüchtern und deutlich Bilanz ziehen. Vergleiche auch Michelsen 2013 und Walter 2013. Zur „unpolitischen Demokratie“ und den Problemen der Repräsentation auch Urbinati 2010.

9 Hierzu jetzt differenziert und kritisch Marg u. a. 2013. 
weis für die Gültigkeit und praktische Relevanz demokratischer Werte und Normen verstanden werden können, die aber gleichzeitig das weitgehend entpolitisierte, von angeblich alternativlosen Imperativen geleitete und vor allem nach Kriterien der marktwirtschaftlichen Effizienz organisierte Management gesellschaftlicher Belange nicht wesentlich behindern" (ebd.: 135).

Blühdorns These fasziniert und beunruhigt gleichermaßen. Der paradoxe Befund der Inanspruchnahme und Entpflichtung von demokratischen Normen öffnet den Blick auf die Ambiguitäten aktueller gesellschaftlich-politischer Entwicklungen. Zugleich stimmt die diagnostizierte Ausweglosigkeit - aus Paradoxien gibt es kein Entrinnen, nur Bewältigungsstrategien - fatalistisch. Doch scheint die ,simulative Demokratie" ein äußerst labiles Gebilde zu sein, welches seine Stabilität einzig der Aufrechterhaltung ihrer Simulationsfähigkeit, des „Als-Ob“, verdankt. Damit ist das Schicksal der „Postdemokratie“ keineswegs besiegelt. Zum einen erzeugt gerade das Widerspiel von demokratischen Grundnormen und davon abweichender politischer Praxis ein Spannungsfeld, welches in sich immer die Möglichkeit grundlegender, auch radikaler Veränderung birgt. Das „,demokratische Versprechen“ lässt sich nicht auf Dauer lahm legen, es ist Movens demokratischer Erneuerung. Zum anderen zeigt die historische Erfahrung, dass Demokratien lernfähige Systeme sind, die sich ihrer veränderten gesellschaftlichen Umwelt anpassen und dabei transformative Kraft entfalten. Insofern ist das „demokratische Projekt“ auch nie abgeschlossen.

Wir haben es in der gegenwärtigen Krisenlage vor allem mit einem Problem zunehmender Entfremdung von politisch-administrativem, (massen-)medialem und wirtschaftlichem System auf der einen Seite und gesellschaftlichen Lebenswelten auf der anderen Seite zu tun. Gesellschaftliche Autonomie hier und institutionelle Abkoppelung dort setzen das repräsentative System einem Stresstest aus. Beides greift nicht ineinander, weil es an intermediären Strukturen der "Transmission“ fehlt, die verlässliche Aggregation von Bürgerinteressen und responsive Entscheidungsfindung der institutionalisierten Politik ermöglichen. Angesichts eines solchen Befundes kann die Zukunft der repräsentativen Demokratie nur in Form von - drei - möglichen Szenarien umschrieben werden. ${ }^{10}$

Da ist erstens die reine Elitendemokratie, in der Funktionsträger und Interessenten eine oligarchisch zu nennende Herrschaft der Wenigen etablieren und Teilhabe zu einem closed shop der Durchsetzungsstarken wird. Der Bürger wird - jenseits des Wahlaktes - allenfalls zur Akklamation von hinter den öffentlichen Kulissen getroffenen Entscheidungen benötigt oder aber zum Stillhalten genötigt. Das politi-

10 Im Folgenden nur leicht veränderte Übernahme aus Vorländer 2011 a sowie 2011 b. 
sche System gibt einerseits den funktionalen Entscheidungsträgern Freiräume politischer Dezision und beschafft sich andererseits Massenloyalität durch einen hohen Output an sozialen Leistungen und Ad hoc-Reaktionen, die auf Stimmungslagen reagieren und Erregungen zu beruhigen suchen. Auf Dauer aber wird sich ein solches verselbständigtes Entscheidungssystem nicht halten können. Zum einen züchtet es Erwartungen, die es schon aus Gründen der Knappheit finanzieller Ressourcen nicht befriedigen kann. Dann macht es sich von den sich beschleunigenden Zyklen medialer Aufmerksamkeit und Kampagnen abhängig. Und schließlich lässt sich, wo formal der Anschein demokratischer Partizipationschancen aufrechterhalten wird, das Teilhabebegehren der Bürger auf Dauer nicht unterdrücken. Aus dem Gefühl von Ohnmacht entwickeln sich Wut und die Bereitschaft zur Rebellion, womöglich auch zu neuem nachhaltigen Engagement. Eruptionen wie „Stuttgart 21“ sind nur verständlich vor dem Hintergrund der Wahrnehmung des repräsentativdemokratischen Systems als geschlossene Veranstaltung von Funktionseliten, wirtschaftlichen Interessenten und eines politisch-bürokratischen Komplexes.

Im zweiten Szenario öffnet sich die repräsentativ verfasste Demokratie der populistischen Versuchung. Vielfach hat die repräsentative Demokratie die Balance zwischen ihren beiden Strukturprinzipien, zwischen dem politischen Entscheidungssystem und ihrer demokratischen Lebensform, verloren und damit eine Wahrnehmung unter den Bürgern erzeugt, wonach die Repräsentanten in wachsender Distanz zu den Repräsentierten agieren. In dieser Differenzerfahrung erzeugen Semantiken der Entfremdung populistische Reaktionsmuster, die die Schließung des Grabens zwischen Politik und Bürger über eine unmittelbare, scheinbar „,unverfälschte" Artikulation des Bürger- und Volkswillens versprechen. Der Populist hört auf die „Stimme des Bürgers“ und tut, „,was der Bürger will“. Die Form unmittelbarer Kommunikation tritt an die Stelle eines diskursiven, über die Foren der Zivilgesellschaft und die Institutionen der repräsentativen Demokratie vermittelten Willens- und Entscheidungsbildung. Die schleichende populistische Metamorphose wird durch die Symbiose von Politik und Medien erheblich begünstigt. Die modernen, audiovisuellen Massenmedien verlangen eine unmittelbare Präsenz, die Tatund Führungskraft suggeriert und auf öffentliche Erregungszustände zu reagieren weiß. Weltweite Kommunikation und beschleunigter Themen- und Agendawechsel prämieren die mediale Performance und suggerieren mit der perfekten Inszenierung bereits die Problembewältigung. Diese virtuelle Unmittelbarkeit des Politischen aber delegitimiert die mediatisierten, in den Institutionen und Verfahren der repräsentativen Demokratie sich vollziehenden komplexen Entscheidungsprozesse vollends. Der Populismus wird zum langen Schatten der ,präsentativen“ Demokratie (Sarcinelli), die zudem immer stärker ihre Verankerung in festgefügten sozialmo- 
ralischen Milieus verliert und sich der Verführungskraft populistischer, charismatischer Führungsfiguren öffnet.

Schließlich das dritte Szenario, das für die Demokratie günstigste: Die repräsentative Demokratie nimmt verstärkt direkte Formen der Beteiligung von Bürgern auf und erhöht die Responsivität des politischen Entscheidungssystems. Eine zugleich responsive und partizipatorische Demokratie verschränkt demokratische Lebensform und demokratische Entscheidungsstruktur auf verschiedenen Ebenen und in verschiedenen Arenen, ohne auf repräsentative Entscheidungsstrukturen zu verzichten. Die Beteiligung von Bürgern in transparenten, kommunalen Planverfahren gehört hier ebenso zur partizipativen Infrastruktur wie förmliche direktdemokratische Verfahren und Runde Tische, in denen Kompromisse mit Beteiligten ausgehandelt, aber nicht mit parteipolitisch und parlamentarisch organisierten Mehrheiten entschieden werden. Verfahren dieser Art erhöhen mit den Beteiligungschancen auch bürgerschaftliche Entscheidungs- und Folgenverantwortung. Sie weichen indes das Monopol repräsentativ-demokratischer Entscheidungsbildung auf und etablieren eine Formenvielfalt zivilgesellschaftlicher Konfliktregulierungsmechanismen. Ein anderer Weg des Bürgers, sich Gehör zu verschaffen, ist darüber hinaus auch der über die Gerichte und dort, wo vorhanden, auch über Verfassungsgerichte und individuelle Verfassungsbeschwerden. Längst gehören Klagen vor Gerichten zu allgemein akzeptierten bürgerschaftlichen Praktiken, was die Judikative mehr und mehr in die allgemeinen politischen Prozesse hineinzieht. Hier wird die Justiz zu einer, in den Formen des Rechts prozedierenden, Arena des Politischen. Wenn kehrseitig vom repräsentativen politischen Entscheidungssystem nun seinerseits erhöhte Responsivität erwartet wird, dann bedeutet das für das politische Personal nicht nur, wie auch populistische Anwandlungen nahelegen würden, ,mit dem Ohr am Puls der Zeit" zu sein. Ansprechbarkeit, Antwortbereitschaft und Reaktionsfähigkeit (Uppendahl 1981) sind vielmehr als Bestandteile eines neuen Führungsverständnisses zu verstehen, das die für notwendig erachteten Entscheidungen öffentlich zu rechtfertigen weiß. Dabei bedeutet Responsivität keineswegs, dass Entscheidungen immer im Einklang mit den Präferenzen der Bürger gefällt werden, zumal diese selbst stark pluralisiert und fragmentiert sind. Aber „,unpopuläre“ Entscheidungen müssen vom Bürger verstanden werden können, auch wenn er ihnen nicht hätte zustimmen wollen oder können. Insgesamt also ist die responsive und partizipative Demokratie ein gemischtes Regime, das über die Gewaltenteilungsund Kontrollsysteme der traditionellen Repräsentativdemokratie weit hinaus geht und demokratischen Entscheidungsprozess und demokratische Lebensform in vielfältiger Weise wieder miteinander zu verkoppeln sucht. 


\section{Literatur}

Beck, Ulrich (Hrsg.), 1998: Politik der Globalisierung. Frankfurt a. M.

Blühdorn, Ingolfur, 2013 a: Simulative Demokratie. Neue Politik nach der postdemokratischen Wende, Berlin.

Blühdorn, Ingolfur, 2013 b: „Das etablierte Lamento trägt nicht zur Veränderung bei““. Das Konzept der simulativen Demokratie. Ein Gespräch mit Ingolfur Blühdorn, in: INDES, Zeitschrift für Politik und Gesellschaft 3, 131-141.

Crouch, Colin, 2008: Postdemokratie, Frankfurt a. M.

Dörner, Andreas, 2001: Politainment. Politik in der medialen Erlebnisgesellschaft, Frankfurt a. M.

Höreth, Marcus, 2009: Überangepasst und realitätsentrückt? Zur Paradoxie der Theorie der deliberativen Demokratie in der EU, in: Zeitschrift für Politikwissenschaft 19 (3), 307-330.

Marg, Stine/Geiges, Lars/Butzlaff, Felix/Walter, Franz, 2013: Die neue Macht der Bürger. Was motiviert die Protestbewegungen? Reinbek bei Hamburg Michelsen, Danny, 2013: Ist das Politische noch zu retten? Eine Bestandsaufnahme mit Hannah Arendt, in: INDES, Zeitschrift für Politik und Gesellschaft 3, 122-130.

Michelsen, Danny/Walter, Franz, 2013: Unpolitische Demokratie. Zur Krise der Repräsentation, Berlin.

Mouffe, Chantal, 2008: Über das Politische, Frankfurt a. M.

Urbinati, Nadia, 2010: Unpolitical Democracy, in: Political Theory 38 (1), 65-92.

Rancière, Jacques, 2002: Das Unvernehmen. Politik und Philosophie, Frankfurt a. M.

Rosanvallon, Pierre, 2006: La contre-démocratie. La politique à l'age de la defiance, Paris.

Uppendahl, Herbert, 1981: "Responsive Demokratie", in: Dietrich Thränhardt/ Herbert Uppendahl (Hrsg.), Alternativen lokaler Demokratie, Königstein i. Ts. Vorländer, Hans, 2011 a: Spiel ohne Bürger, Frankfurter Allgemeine Zeitung, 12. Juli 2011, 8.

Vorländer, Hans, 2011 b: Der Wutbürger. Repräsentative Demokratie und kollektive Emotionen, in: Harald Bluhm/Karsten Fischer/Marcus Llanque (Hrsg.), Ideenpolitik. Geschichtliche Konstellationen und gegenwärtige Konflikte, Berlin.

Walter, Franz, 2013: Gefahren der Demokratie. Skeptische Betrachtungen zur Lage, in: INDES, Zeitschrift für Politik und Gesellschaft 3, 110-121. 
Zürn, Michael, 1998: Regieren jenseits des Nationalstaates. Globalisierung und Denationalisierung als Chance, Frankfurt a. M.

Korrespondenzanschrift:

Prof. Dr. Hans Vorländer

Lehrstuhl für Politische Theorie und Ideengeschichte

Institut für Politikwissenschaft und Zentrum für Verfassungs- und

Demokratieforschung

TU Dresden

01062 Dresden

E-Mail: hans.vorlaender@tu-dresden.de 